\title{
Identification of the State of Maximal Hyperemia in the Assessment of Coronary Fractional Flow Reserve Using Non-Invasive Electrical Velocimetry
}

\author{
Takahide Murasawa, ${ }^{1 *}$, Masao Takahashi, ${ }^{2 *} \mathrm{MD}$, Masahiro Myojo, ${ }^{2} \mathrm{MD}$, \\ Arihiro Kiyosue, ${ }^{2} \mathrm{MD}$, Atsushi OGURI, ${ }^{2} \mathrm{MD}$, Jiro ANDO, ${ }^{2} \mathrm{MD}$, and Issei Komuro, ${ }^{2} \mathrm{MD}$
}

\begin{abstract}
SUMMARY
Previous research revealed that, in patients with coronary pressure-derived fractional flow reserve (FFR) in the 'grey zone' (0.75-0.85), repeated FFR assessments sometimes yield conflicting results. One of the causes of the fluctuations in FFR values around the grey zone may be imprecise identification of the point where maximal hyperemia is achieved. Identification of the state of maximal hyperemia during assessment of FFR can be challenging. This study aimed to determine whether non-invasive electrical velocimetry (EV) can be used to identify the state of maximal hyperemia.

Stroke volume (SV), SV variation (SVV), and systemic vascular resistance index (SVRI) were determined by EV in 15 patients who underwent FFR assessment. Time intervals from initiation of adenosine infusion to achieving maximal hyperemia (time ${ }^{\mathrm{mFRR}}$ ), as well as to achieving maximal cardiac output (CO), SV, SVV, and SVRI (time ${ }^{\mathrm{mCO}}$, time ${ }^{\mathrm{mSV}}$, time $^{\mathrm{mSVV}}$, and time ${ }^{\mathrm{mSVRI}}$, respectively), were determined. Time ${ }^{\mathrm{mCO}}$ and time $\mathrm{mSVV}^{\mathrm{mSV}}$ were closer to time ${ }^{\mathrm{mFRR}}$ than other values (time $^{\mathrm{mSVV}} /$ time $^{\mathrm{mFRR}}$ versus time $\mathrm{mSVR}^{\mathrm{mSt} / \mathrm{time}} \mathrm{mFRR}^{\mathrm{mFR}}=1.03 \pm 0.2$ versus $\left.1.36 \pm 0.4, P<0.05\right)$. The maximum of $\mathrm{SV}$ was difficult to determine owing to considerable variations, but the maximum of SVV was clearly recognized. Time ${ }^{\mathrm{mCO}}$ and time ${ }^{\mathrm{mSVV}}$ were significantly correlated with time ${ }^{\mathrm{mFFR}}$, with time ${ }^{\mathrm{mSVV}}$ showing a stronger correlation than time ${ }^{\mathrm{mSV}}\left(\mathrm{time}^{\mathrm{mSVv}}: r=0.92\right.$, $P<0.01 ;$ time $\left.^{\mathrm{mCO}}: r=0.80, P<0.01\right)$.
\end{abstract}

Maximal SVV is reached close to maximal hyperemia. Monitoring of SVV with non-invasive EV during FFR assessment can help identify the state of maximal hyperemia. (Int Heart J 2017; 58: 365-370)

Key words: Stroke volume variation, Coronary heart disease, Systemic vascular resistance, $\mathrm{Pd} / \mathrm{Pa}$

$\mathrm{C}$ oronary pressure-derived fractional flow reserve (FFR), which is determined as the ratio of distal to proximal pressure $(\mathrm{Pd} / \mathrm{Pa})$ under conditions of maximal hyperemia, represents a reproducible indicator of physiologically significant coronary stenosis. ${ }^{1,2)}$ FFR-guided percutaneous coronary intervention (PCI) has significantly better clinical outcomes than PCI guided by angiography. ${ }^{3,4)}$ Therefore, therapy guided by FFR is a key part of clinical management in patients with coronary artery disease (CAD).

Although maximal hyperemia is a crucial factor for the assessment of FFR, ${ }^{5)}$ it is not always easy to obtain confirmatory evidence of reaching the maximal point of hyperemia in clinical practice. Intravenous adenosine administration is considered the gold standard for elicitation of maximal hyperemia, ${ }^{3)}$ but there is no clear evidence indicating the point of maximal hyperemia. Moreover, heart rate and blood pressure fluctuate because of respiration and are influenced by intravenous adenosine administration itself, which may lead to an inaccurate FFR. Intravenous adenosine administration is known to increase myocardial blood flow, cardiac output $(\mathrm{CO})$, and stroke volume (SV) and to reduce systemic vascular resistance index (SVRI) ${ }^{6,7)}$ Maximal hyperemia is a condition of maximal vasodilatation with the highest SV and the lowest SVRI. Accordingly, the monitoring of SV and SVRI may be used to verify whether maximal hyperemia has been achieved. Although pulmonary artery catheterization (PAC) is a reliable and reproducible method for assessing circulatory dynamics, ${ }^{8}$ $\mathrm{CO}$ and SV measured with non-invasive electrical velocimetry (EV) were recently shown to be a viable alternative. ${ }^{9,10}$ Noninvasive $\mathrm{EV}$ is based on impedance cardiography, which can measure fluctuations in aortic blood volume during the cardiac cycle. EV additionally traces the direction and changes in the mechanical characteristics of erythrocytes for circulatory dynamics calculation. In the beginning of systole, this orientation changes into an alignment that leads to an acceleration of the erythrocytes, causing a maximum rate of change in electrical impedance. The general condition during FFR assessment is relatively steady, with intravenous adenosine administration

From the ${ }^{1}$ Department of Medical Engineering, The University of Tokyo Hospital and ${ }^{2}$ Department of Cardiovascular Medicine, Graduate School of Medicine, The University of Tokyo, Tokyo, Japan.

"These authors contributed equally to this work.

Address for correspondence: Masao Takahashi, MD, Department of Cardiovascular Medicine, Graduate School of Medicine, The University of Tokyo, 7-3-1 Hongo, Bunkyo-ku, Tokyo 113-8655, Japan. E-mail: masaotakahashi-gi@umin.org

Received for publication September 25, 2016. Revised and accepted October 28, 2016.

Released in advance online on J-STAGE May 23, 2017.

All rights reserved by the International Heart Journal Association. 
being the most significant affecting factor. Therefore, it is feasible to monitor circulatory dynamics using EV during FFR.

The purpose of this investigation was to determine whether non-invasive EV can provide supporting evidence for reaching the state of maximal hyperemia.

\section{Methods}

Study design and patient population: This study was a singlecentre, pilot, retrospective, observational study designed to investigate the relationship between maximal hyperemia and circulatory dynamics using EV. The present study enrolled consecutive patients with CAD who underwent the FFR assessment at the Department of Cardiovascular Medicine, The Tokyo University Hospital (Tokyo, Japan), from December 2013 to July 2015. The criteria for exclusion included prior cardiac bypass grafting surgery, cardiac valve disease, severe low cardiac function (ejection fraction $<30 \%$ ), arrhythmia, including atrial fibrillation, unstable vital signs during the FFR assessment, non-recorded minimum $\mathrm{Pd} / \mathrm{Pa}$ ratio, and insufficient $\mathrm{EV}$ recording. Informed consent was obtained from each participant or the participant's legal representative. The study protocol, which adhered to the principles of the Declaration of Helsinki, was approved by The University of Tokyo's Institutional Review Board. Fifteen patients with a mean age of 71.1 \pm 10.0 years, who needed an assessment of FFR, were enrolled.

Coronary angiography with FFR assessment: Coronary angiography was performed via the radial or femoral percutaneous approach. The degree of coronary artery stenosis was rated visually using a caliper by two experienced interventional cardiologists. Angiography, which revealed moderate coronary stenosis of $50-75 \%$ in all patients, was followed by the assessment of FFR. FFR measurements were performed as previously described. ${ }^{11)}$ Briefly, a 0.014 -inch pressure monitoring guidewire (Pressure Wire, Radi Medical Systems, St. Jude Medical, MN, USA) was calibrated and introduced into the guiding catheter. After the aortic guiding catheter pressure and the wire tip pressure became equal, the wire was advanced distally to the target coronary stenosis. FFR was calculated as the ratio of distal coronary pressure $(\mathrm{Pd})$ to aortic pressure $(\mathrm{Pa})$ obtained after the achievement of maximal hyperemia elicited by intravenous adenosine administration at a rate of $160 \mu \mathrm{g}$ per kilogram of body weight per minute through a central vein (RadiAnalyzer, St. Jude Medical). The point with the lowest $\mathrm{Pd} / \mathrm{Pa}$ ratio was considered as the state of maximal hyperemia. When this lowest point was recognized, we injected contrast medium, which causes vasodilatation known as reactive hyperemia, in the artery to ensure that the $\mathrm{Pd} / \mathrm{Pa}$ ratio remained constant. ${ }^{12)} \mathrm{A} \mathrm{Pd} / \mathrm{Pa}$ ratio of 0.80 was considered the significant ischemic threshold. ${ }^{3)}$ The time from initiation of intravenous adenosine infusion to reaching the state of maximal hyperemia, defined as the lowest value of $\mathrm{Pd} / \mathrm{Pa}$, was recorded $\left(\right.$ time $\left.^{\mathrm{mFRR}}\right)$.

EV monitoring: To evaluate cardiac function and dynamics, EV was performed during the measurement of FFR using the transthoracic electrical bioimpedance technique (Aesculon, Osypika Medical, Berlin, Germany). Electrodes were placed at 4 points as previously described, and the Bernstein-Osypka equation was used for calculating $\mathrm{CO}, \mathrm{SV}, \mathrm{SV}$ variation (SVV), and SVRI. ${ }^{910)}$ SVV was calculated as follows:

$\mathrm{SVV}=$ standard deviation $(\mathrm{SD})$ of SV / mean SV $\times 100(\%)$

Time intervals from initiation of intravenous adenosine infusion to reaching extremums (maximums or minimums) of $\mathrm{CO}, \mathrm{SV}, \mathrm{SVV}$, and SVRI, as determined using EV, were also recorded $\left(\right.$ time $^{\mathrm{mCO}}$ time $^{\mathrm{mSV}}$, time ${ }^{\mathrm{mSVV}}$, and time ${ }^{\mathrm{mSVRI}}$, respectively). Baseline haemodynamic parameters were recorded at least 2 minutes before intravenous adenosine infusion in the absence of any stimulation or administration of medical substances.

Statistical analysis: Data are expressed as the mean \pm SD. Differences in continuous variables between 2 groups were compared using an unpaired $t$-test when the variables showed a normal distribution. Differences in continuous variables within a group were compared using paired $t$-tests when the variables showed a normal distribution and Wilcoxon's signed-rank test when the variables were not normally distributed. Categorical variables were compared between 2 groups using Fisher's exact test. The Pearson correlation test was performed to identify the factors that correlated with time ${ }^{\mathrm{mFFR}}$ and time $\mathrm{mSVV}^{\mathrm{mSV}}$. These calculations were performed using JMP, version 11.2 (SAS Institute Inc., Cary, NC, USA). All analyses were two-sided, and a $P$-value of less than 0.05 was considered to indicate statistical significance.

\section{Results}

Fifteen patients who underwent FFR assessment were included in this study. The clinical characteristics of the patients are shown in Table I. The patients tended to be middle-aged and predominantly male, and moderate stenosis was observed mostly in the left anterior descending artery. The baseline and maximal or minimal values of the parameters obtained during FFR and EV assessments are shown in Table II. The SV, CO, and SVV were significantly increased, whereas the $\mathrm{Pd} / \mathrm{Pa}$ ratio and SVRI were significantly reduced by intravenous adenosine administration. These variations were consistent with the findings of previous reports. ${ }^{6,7)}$ The times of reaching the extremums are also shown in Table II. The values of time ${ }^{\mathrm{mSVV}}$ were

Table I. Basic Clinical Characteristics of the Patients

\begin{tabular}{lc}
\hline Variable, $n=15$ & Average \pm SD or numbers \\
\hline Male, $\%$ & $10(66.7 \%)$ \\
Age, years & $71.1 \pm 10.0$ \\
BW, kg & $62.5 \pm 14.4$ \\
BMI, kg/m ${ }^{2}$ & $24.1 \pm 4.0$ \\
Systolic BP, mmHg & $125.0 \pm 16.9$ \\
Diastolic BP, mmHg & $65.0 \pm 12.0$ \\
LVEF, $\%$ & $61.1 \pm 9.9$ \\
Number of vessel disease & $1.7 \pm 0.62$ \\
Single & 6 \\
Double & 8 \\
Triple & 1 \\
FFR measured artery & \\
LAD & 12 \\
LCX & 0 \\
RCA & 3 \\
\hline
\end{tabular}

BW indicates body weight; BMI, body mass index; BP, blood pressure; LVEF, left ventricular ejection fraction; FFR, fractional flow reserve; LAD, left anterior descending artery; LCX, left circumflex artery; and RCA, right coronary artery. 
Table II. FFR Values and the Results of EV Measurements

\begin{tabular}{lccc}
\hline Variable, $n=15$ & Baseline & Maximum/Minimum point & $P^{*}$ \\
\hline FFR & $0.93 \pm 0.03$ & $0.83 \pm 0.07$ & $<0.01$ \\
CO, $\mathrm{L} /$ minute $/ \mathrm{m}^{2}$ & $3.61 \pm 1.16$ & $5.27 \pm 1.91$ & $<0.01$ \\
SV, $\mathrm{mL}$ & $47.58 \pm 15.28$ & $70.19 \pm 19.84$ & $<0.01$ \\
SVV, $\%$ & $5.07 \pm 1.75$ & $19.13 \pm 3.09$ & $<0.01$ \\
SVRI, dynes·seconds $/ \mathrm{cm}^{5} / \mathrm{m}^{2}$ & $3077.3 \pm 1249.7$ & $2036.7 \pm 812.1$ & $<0.01$ \\
Time at peak point, seconds & & & - \\
FFR & - & $183.6 \pm 81.6$ & - \\
CO & - & $214.3 \pm 88.5$ & - \\
SV & - & $216.3 \pm 94.0$ & - \\
SVV & - & $181.5 \pm 87.3$ & - \\
SVRI & - & $239.2 \pm 83.1$ & \\
\hline
\end{tabular}

FFR indicates fractional flow reserve; EV, electrical velocimetry; $\mathrm{CO}$, cardiac output; SV, stroke volume; SVV, stroke volume variation; SVRI, systemic vascular resistance index. $P^{*}$ : The variables are compared with respect to their baselines and peak points.

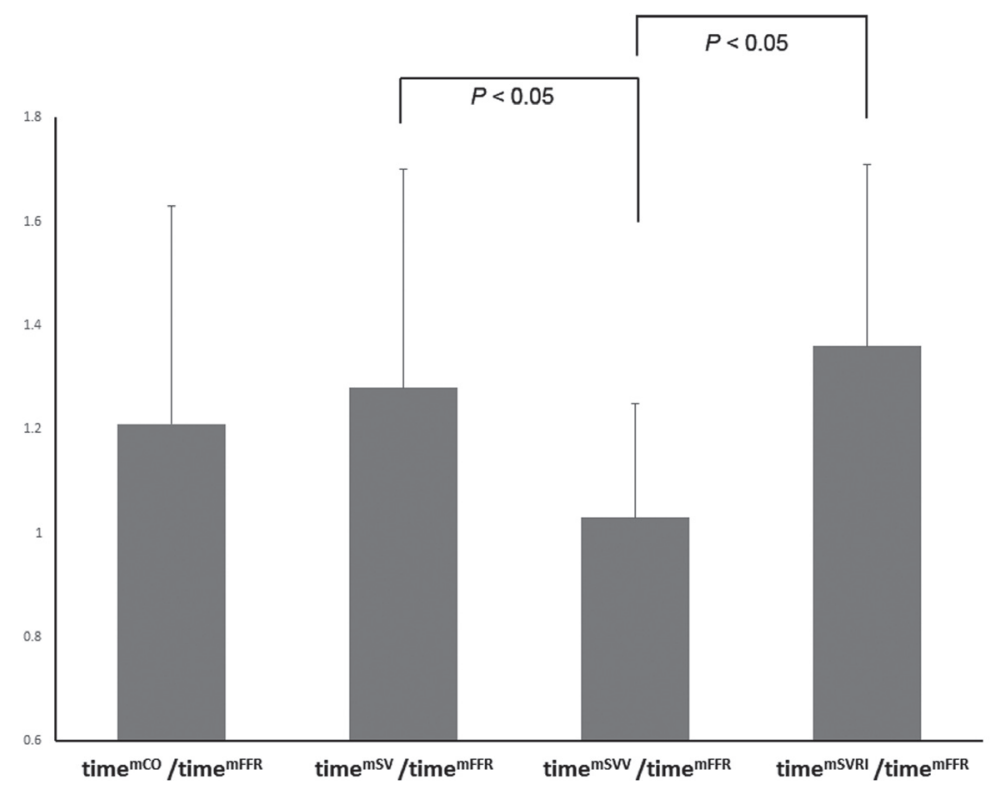

Figure 1. Comparison of the time points of peaks in cardiac output (CO), stroke volume (SV), stroke volume variation (SVV), and systemic vascular resistance index (SVRI) to the time points of peaks of the Pd/Pa ratio in fractional flow reserve (FFR). The ratio of the time of maximal SVV $\left(t_{i m e} \mathrm{mSVV}^{\mathrm{mSV}}\right.$ ) to the time of maximal FFR (time $\left.{ }^{\mathrm{mFFR}}\right)$ was close to 1.0. The ratio of time $\mathrm{m}^{\mathrm{mSV}}$ to time $\mathrm{m}^{\mathrm{mFR}}$ was significantly different from the ratios of the time of maximal SV (time $\mathrm{m}^{\mathrm{mSV}}$ ) and SVRI $\left(\right.$ time $\left.^{\mathrm{mSVRI}}\right)$ to time $\mathrm{m}^{\mathrm{mFR}}$, whereas there was no significant difference in the ratio of the time of maximal $\mathrm{CO}\left(\mathrm{time}^{\mathrm{mCO}}\right)$ to time $^{\mathrm{mFFR}}$.

closest to the values of time ${ }^{\mathrm{mFFR}}$. Therefore, we compared all time intervals after normalizing by time ${ }^{\mathrm{mFFR}}$ (Figure 1). Normalized time ${ }^{\mathrm{mSVV}}$ was significantly closer to 1.0 than normalized time $^{\mathrm{mSV}}$ and time ${ }^{\mathrm{mSVRI}}$, whereas there was no significant difference between normalized time $\mathrm{mCO}^{\mathrm{mCO}}$ and normalized time $^{\mathrm{mSVV}}\left(\mathrm{time}^{\mathrm{mCO}} / \mathrm{time}^{\mathrm{mFFR}}\right.$ versus time ${ }^{\mathrm{mSVV}} / \mathrm{time}^{\mathrm{mFFR}}=1.21 \pm$ 0.44 versus $1.03 \pm 0.22, P=0.08$ ). These results suggested that the points of maximal SVV or CO could represent the point of maximal hyperemia. Next, time ${ }^{\mathrm{mSVV}}$ and time ${ }^{\mathrm{mCO}}$ were evaluated for correlations with time ${ }^{\mathrm{mFFR}}$. As shown in Figure 2, time $^{\mathrm{mSVV}}$ correlated more strongly with time ${ }^{\mathrm{mFFR}}$ than time ${ }^{\mathrm{mCO}}$ (time $^{\text {mSVV }}$ versus time ${ }^{\text {mFFR }}: r=0.92$; time ${ }^{\text {mCO }}$ versus time ${ }^{\text {mFFR }}: r$ $=0.80$ ). The time courses of CO, SV, and SVRI obtained by EV did not display clear peaks, whereas prominent peaks were recorded for SVV (Figure 3 shows the raw data for case 1). Moreover, time ${ }^{\mathrm{mFFR}}$ (115.77 seconds) was close to the time cor- responding to the peak of SVV (120.05 seconds). Based on these results, SVV determined with EV could be used to confirm achieving maximal hyperemia. Furthermore, one of the cases in our study showed that the $\mathrm{Pd} / \mathrm{Pa}$ ratio fluctuated and there were 2 competing peaks in the $\mathrm{Pd} / \mathrm{Pa}$ ratio under intravenous adenosine administration (Figure 4). The minimal peak of the $\mathrm{Pd} / \mathrm{Pa}$ ratio was recognized eventually as 0.83 at 251 seconds after the initiation of intravenous adenosine administration, whereas simultaneous EV record showed that the maximal peak of SVV can be clearly confirmed at the same time around the minimal peak of the $\mathrm{Pd} / \mathrm{Pa}$ ratio with more than 2.5fold increase. These data suggested a peak of SVV with more than a 2.5-fold increase might be useful supporting evidence to recognize the minimal $\mathrm{Pd} / \mathrm{Pa}$ ratio. 

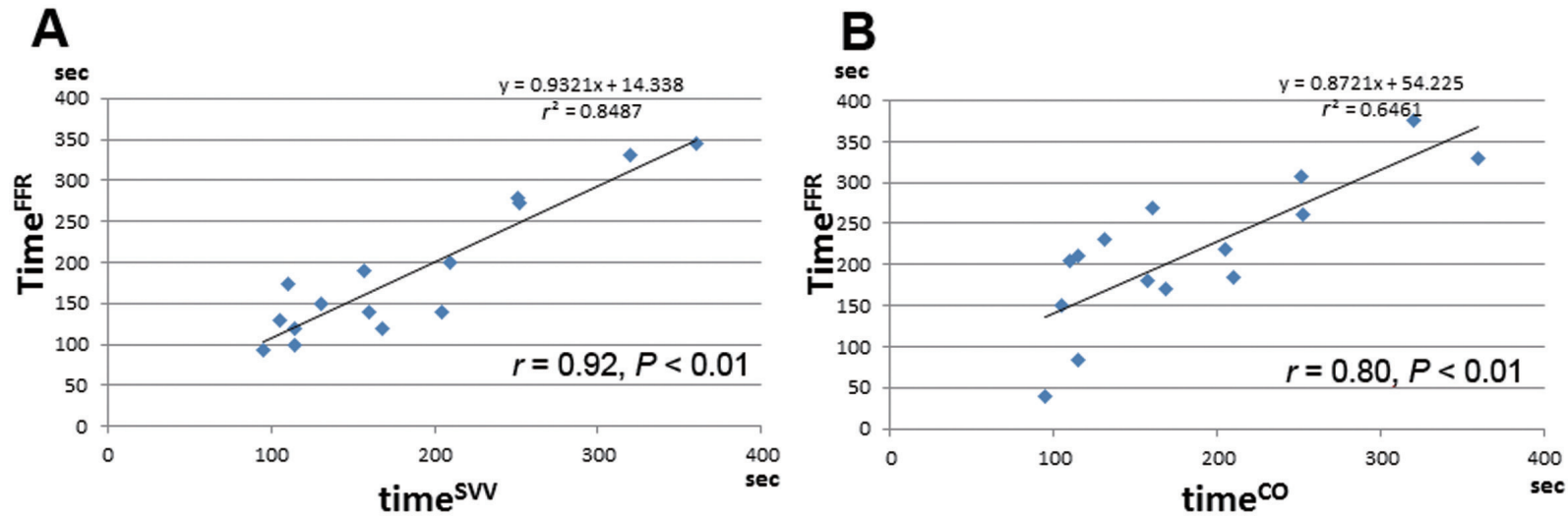

Figure 2. A: Correlation between the positions of peaks of the Pd/Pa ratio in fractional flow reserve (FFR, time $\left.{ }^{\mathrm{mFFR}}\right)$ and stroke volume variation $(\mathrm{SVV}$, time $\left.^{\mathrm{mSVV}}\right)$. B: Correlation between time $\mathrm{m}^{\mathrm{mFFR}}$ and the positions of peaks of cardiac output $\left(\mathrm{CO}\right.$, time $\left.^{\mathrm{mCO}}\right)$.

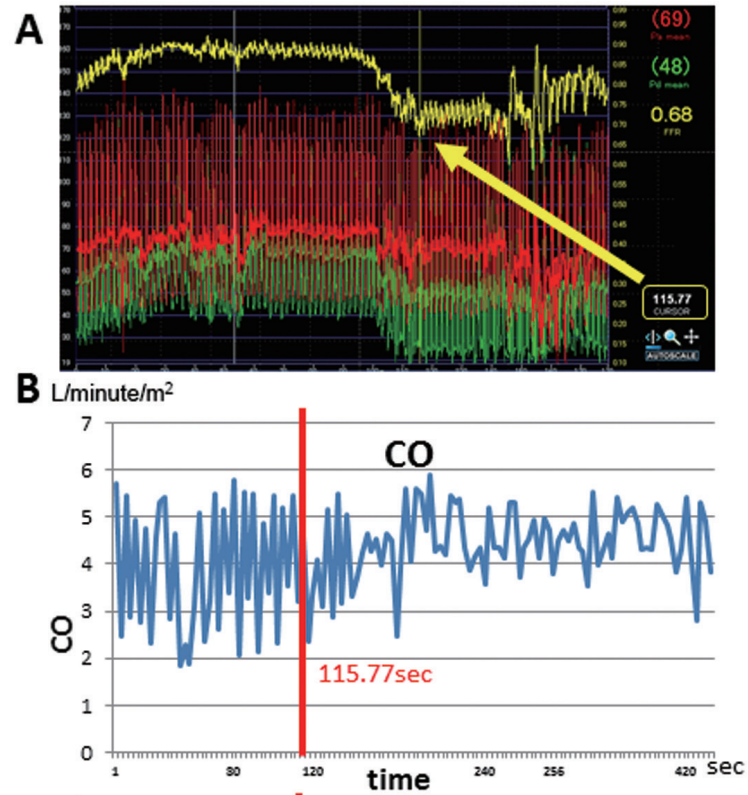

D

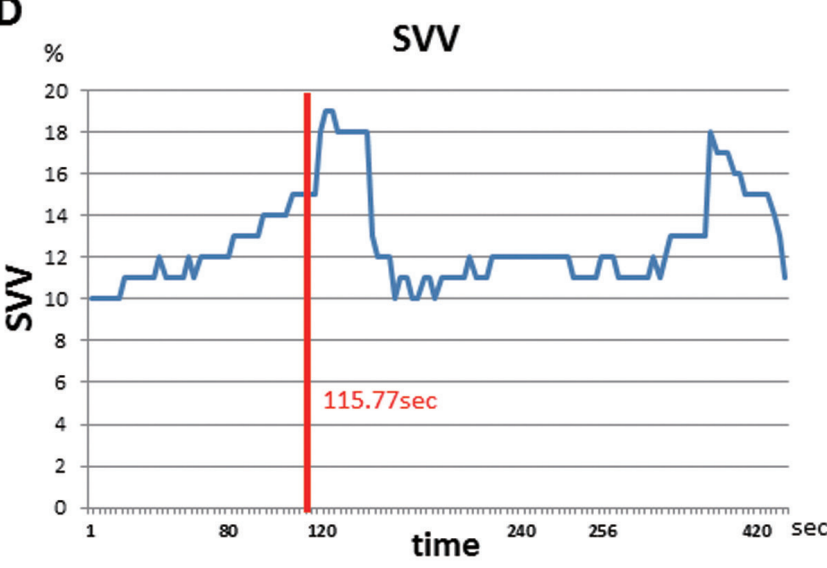

$\mathbf{E}$

dynes" sec/cm5/m²

SVRI

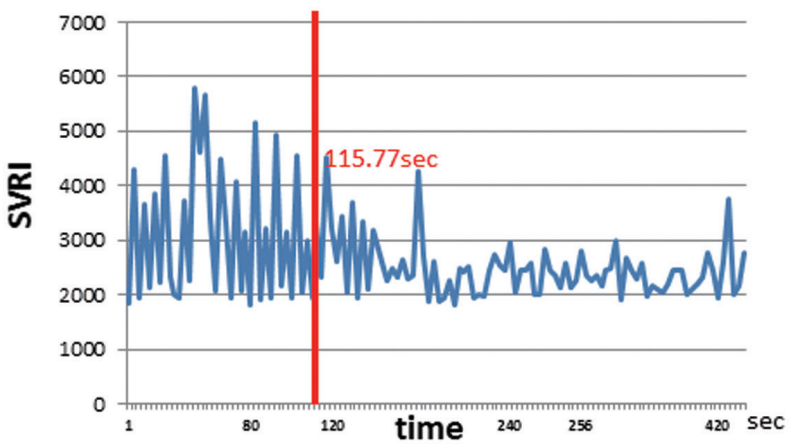

Figure 3. Assessment of fractional flow reserve (FFR) and electrical velocimetry (EV) measurements for a single case. A: The minimal Pd/Pa ratio of 0.68 occurred $115.8 \mathrm{~s}$ after the initiation of intravenous adenosine infusion. B, C, D, and $\mathbf{E}$ present the time plots of cardiac output (CO), stroke volume (SV), stroke volume variation (SVV), and systemic vascular resistance index (SVRI), respectively, which were monitored with EV. The red lines in panels B, C, $\mathrm{D}$, and $\mathrm{E}$ correspond to the minimum of the $\mathrm{Pd} / \mathrm{Pa}$ ratio.

\section{DiscuSsion}

Although FFR, which is determined under maximal hyperemia state, is considered as the physiology standard in the functional assessment of intermediate coronary stenosis, ${ }^{13,14)}$ the state of maximal hyperemia induced by intravenous adenosine administration is somewhat uncertain during the FFR measurement in clinical practice. The availability of approach- 


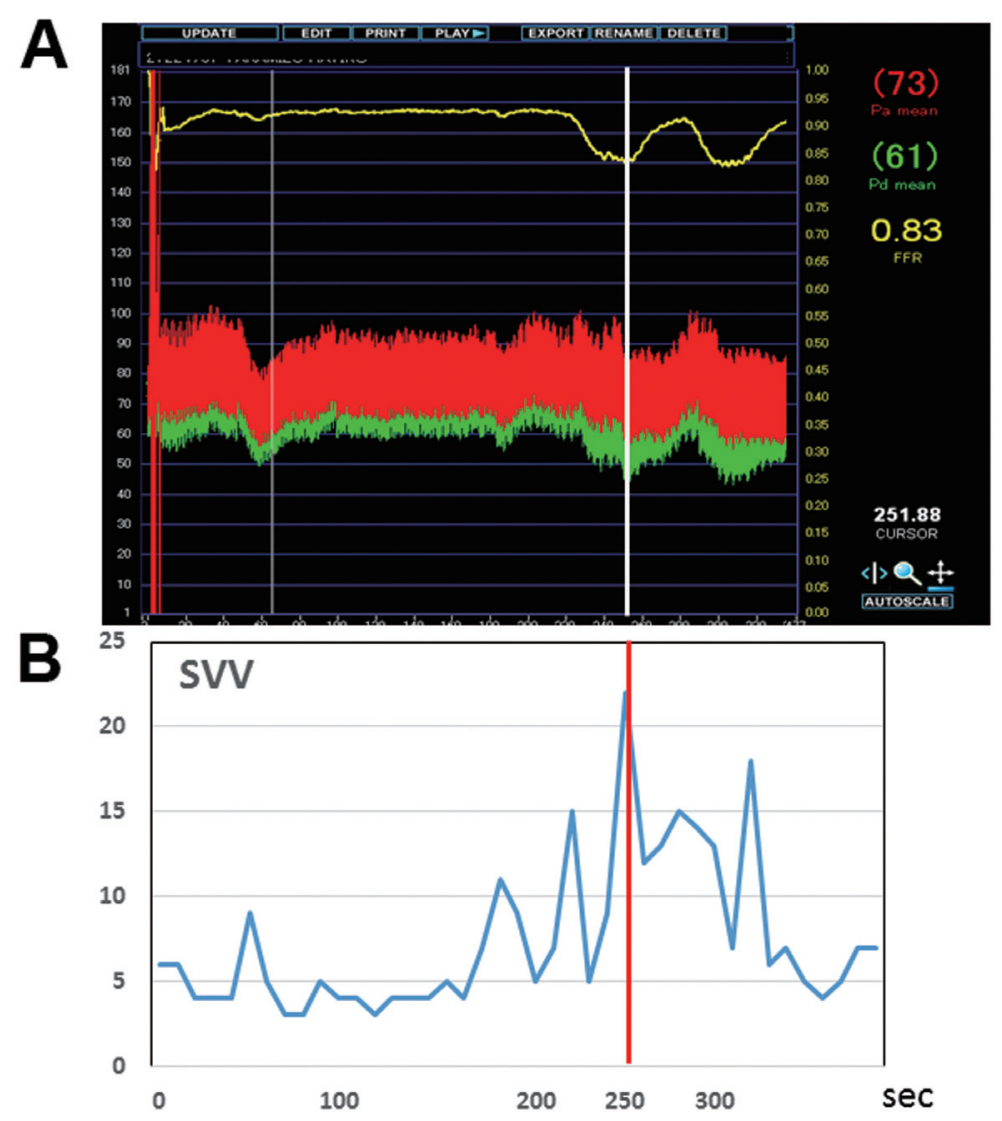

Figure 4. Assessment of fractional flow reserve (FFR) and stroke volume variation (SVV) measurements for a single other case. A: The minimal $\mathrm{Pd} / \mathrm{Pa}$ ratio of 0.83 occurred $251.88 \mathrm{~s}$ after the initiation of intravenous adenosine infusion. B presents the time plots of SVV with simultaneous recording. The maximal peak of SVV occurred $250 \mathrm{~s}$ after the initiation of intravenous adenosine infusion.

es that can confirm the state of maximal hyperemia could increase the accuracy of FFR measurements. In this regard, a previous study revealed that, in patients with a $\mathrm{Pd} / \mathrm{Pa}$ ratio in the 'grey zone' $(0.75-0.85)$, repeated FFR assessments sometimes yield conflicting results. ${ }^{15}$ One of the causes of the fluctuations in FFR values around the grey zone may be imprecise identification of the point where maximal hyperemia is achieved. The main finding of the present analysis is that the peaks of $\mathrm{CO}, \mathrm{SV}$, and SVV detected with EV were in close proximity to the peak of the $\mathrm{Pd} / \mathrm{Pa}$ ratio, which is usually considered to correspond to the state of maximal hyperemia in clinical practice. The peak of SVV was closest to the peak of the $\mathrm{Pd} / \mathrm{Pa}$ ratio, which suggests that SVV is the most useful parameter to determine the point of maximal hyperemia.

Since previous studies showed that myocardial blood flow, $\mathrm{CO}$, and SV responded to intravenous adenosine infusion, ${ }^{6,7)}$ we hypothesized that maximal hyperemia could be detected by monitoring these variables. Assessment of hemodynamic parameters during FFR measurements has not been reported so far. Furthermore, although hemodynamic data can be obtained reliably and reproducibly by invasive techniques such as PAC, it has been recently demonstrated that non-invasive techniques that utilize EV can be successfully employed for this purpose. ${ }^{9,10)}$ However, it was also found that data obtained with EV did not fully correlate with those obtained with
PAC ${ }^{16)}$ because of the impact of arrhythmia, increased thoracic fluid content, and valve disease on the results of EV. ${ }^{17,18)}$ Therefore, we excluded patients with arrhythmia, cardiac shunt, valve disease, and severe heart failure from the present study. $\mathrm{SV}$ and CO measured by EV can also be affected, at least in part, by respiratory artifacts, fractional body motion, and body fluid change, leading to fluctuations in values. However, as the present study shows, patients in a stable state, including stable body fluid balance, can be successfully evaluated using EV.

In the present study, the peaks of SV and CO could not be recognized clearly because SV and CO vary within a heartbeat, and the corresponding maxima were obscured by these repeating variations. In contrast, the curve of SVV, which reflects the rate of change of SV, was smooth, allowing easy detection of peaks (Figure 3). Moreover, intravenous adenosine infusion increased the peak SVV signal more than two-fold, whereas the peaks of $\mathrm{CO}$ and SV were only increased by approximately half. These factors resulted in SVV being the best indicators of the study. SVV has been shown to have very high sensitivity and specificity to volume status and to be a good predictor of hypovolemia and fluid responsiveness. ${ }^{19)}$ High variability of SV indicates the presence of hypovolemia at a steady vascular tone, whereas vasodilators have been shown to increase SVV at constant body fluid. ${ }^{20)}$ The strong correlation between peaks of the $\mathrm{Pd} / \mathrm{Pa}$ ratio and SVV observed in this 
study might reflect the impact of changing vascular tone. Thus, since body fluid volume remains relatively constant during FFR assessment, SVV may represent changes in vascular tone.

The $\mathrm{Pd} / \mathrm{Pa}$ ratio at maximal hyperemia is affected by the degree of stenosis, whereas the positions of SVV peaks are influenced by vascular tone and preload. This precludes direct evaluation of the relations between these two parameters. Instead, we assessed the time points at which the peaks occurred. Limitation: The present study had some limitations. First, although the times of the peaks of SVV closely corresponded to the times of maximal hyperemia, precise detection of the latter required establishing a cut-off point for SVV increase. During the FFR evaluation, we observed a 2.5 -fold increase in SVV near the point of maximal hyperemia in almost all cases. This suggests that a 2.5-fold increase in SVV may indicate the state of maximal hyperemia during FFR measurement. However, further studies are needed to explore the relationship between levels of SVV and maximal hyperemia. Second, because this was a retrospective single-centre analysis, selection bias may not be entirely excluded. Third, owing to the stringent inclusion criteria, the number of subjects was small. Hence, a largescale, multi-centre, prospective study is necessary to confirm the results of this study.

Conclusion: The peaks of SVV obtained by EV are highly correlated with peaks of the $\mathrm{Pd} / \mathrm{Pa}$ ratio, which are thought to represent the state of maximal hyperemia. Therefore, monitoring of SVV with EV could be used to confirm achieving maximal hyperemia.

Impact on daily practice: Monitoring of SVV by EV assessment is safe and easy, and could be used to identify achievement of maximal hyperemia. Thus, when the point of FFR is in the "grey zone", EV can help to confirm whether the point in question is indeed maximal hyperemia.

\section{ACKNOWLEDGMENTS}

The authors thank the Department of Medical Engineering for their help in data acquisition for this study.

\section{Disclosure}

Conflict of interest statement: The authors declare that there are no conflicts of interest.

\section{REFERENCES}

1. Pijls NH, van Son JA, Kirkeeide RL, De Bruyne B, Gould KL. Experimental basis of determining maximum coronary, myocardial, and collateral blood flow by pressure measurements for assessing functional stenosis severity before and after percutaneous transluminal coronary angioplasty. Circulation 1993; 87: 1354-67.

2. Pijls NH, De Bruyne B, Peels K, et al. Measurement of fractional flow reserve to assess the functional severity of coronary-artery stenoses. N Engl J Med 1996; 334: 1703-8.

3. Tonino PA, De Bruyne B, Pijls NH, et al; FAME Study Investigators. Fractional flow reserve versus angiography for guiding percutaneous coronary intervention. N Engl J Med 2009; 360: 21324.
4. Pijls NH, Fearon WF, Tonino PA, et al; FAME Study Investigators. Fractional flow reserve versus angiography for guiding percutaneous coronary intervention in patients with multivessel coronary artery disease: 2-year follow-up of the FAME (Fractional Flow Reserve Versus Angiography for Multivessel Evaluation) study. J Am Coll Cardiol 2010; 56: 177-84.

5. Leone AM, Porto I, De Caterina AR, et al. Maximal hyperemia in the assessment of fractional flow reserve: intracoronary adenosine versus intracoronary sodium nitroprusside versus intravenous adenosine: the NASCI (Nitroprussiato versus Adenosina nelle Stenosi Coronariche Intermedie) study. JACC Cardiovasc Interv 2012; 5: 402-8.

6. González-Alonso J, Mortensen SP, Jeppesen TD, et al. Haemodynamic responses to exercise, ATP infusion and thigh compression in humans: insight into the role of muscle mechanisms on cardiovascular function. J Physiol 2008; 586: 2405-17.

7. Komukai K, Hashimoto K, Shibata T, et al. Effect of continuous ATP injection on human hemodynamics. Circ J 2002; 66: 926-9.

8. Binanay C, Califf RM, Hasselblad V, et al; ESCAPE Investigators and ESCAPE Study Coordinators. Evaluation study of congestive heart failure and pulmonary artery catheterization effectiveness: the ESCAPE trial. JAMA 2005; 294: 1625-33.

9. Petter H, Erik A, Björn E, Göran R. Measurement of cardiac output with non-invasive Aesculon impedance versus thermodilution. Clin Physiol Funct Imaging 2011; 31: 39-47.

10. Schubert S, Schmitz T, Weiss M, et al. Continuous, non-invasive techniques to determine cardiac output in children after cardiac surgery: evaluation of transesophageal Doppler and electric velocimetry. J Clin Monit Comput 2008; 22: 299-307.

11. Pijls NH, Van Gelder B, Van der Voort P, et al. Fractional flow reserve. A useful index to evaluate the influence of an epicardial coronary stenosis on myocardial blood flow. Circulation 1995; 92: 3183-93.

12. Kloster FE, Friesen WG, Green GS, Judkins MP. Effects of coronary arteriography on myocardial blood flow. Circulation 1972; 46: 438-44.

13. Miyazaki T, Ashikaga T, Ohigashi H, Komura M, Kobayashi K, Isobe M. Impact of smoking on coronary microcirculatory resistance in patients with coronary artery disease. Int Heart J 2015; 56: 29-36.

14. Zafar H, Sharif F, Leahy MJ. Feasibility of intracoronary frequency domain optical coherence tomography derived fractional flow reserve for the assessment of coronary artery stenosis. Int Heart $\mathrm{J}$ 2014; 55: 307-11.

15. Petraco R, Sen S, Nijjer S, et al. Fractional flow reserve-guided revascularization: practical implications of a diagnostic gray zone and measurement variability on clinical decisions. JACC Cardiovasc Interv 2013; 6: 222-5.

16. Tomaske M, Knirsch W, Kretschmar O, et al; Working Group on Non-invasive Haemodynamic Monitoring in Paediatrics. Cardiac output measurement in children: comparison of Aesculon cardiac output monitor and thermodilution. Br J Anaesth 2008; 100: 51720.

17. Moshkovitz Y, Kaluski E, Milo O, Vered Z, Cotter G. Recent developments in cardiac output determination by bioimpedance: comparison with invasive cardiac output and potential cardiovascular applications. Curr Opin Cardiol 2004; 19: 229-37. (Review)

18. Doering L, Lum E, Dracup K, Friedman A. Predictors of betweenmethod differences in cardiac output measurement using thoracic electrical bioimpedance and thermodilution. Crit Care Med 1995; 23: 1667-73.

19. Reuter DA, Kirchner A, Felbinger TW, et al. Usefulness of left ventricular stroke volume variation to assess fluid responsiveness in patients with reduced cardiac function. Crit Care Med 2003; 31: 1399-404.

20. Bouchacourt JP, Riva JA, Grignola JC. The increase of vasomotor tone avoids the ability of the dynamic preload indicators to estimate fluid responsiveness. BMC Anesthesiol 2013; 13: 41. 